\title{
REAlistische Betrachtungen
}

Bernhard Gurtner

Korrespondenz:

Dr. med. Bernhard Gurtner

Eggstrasse 76

CH-8620 Wetzikon

gurtner.bernhard@bluewin.ch
Schon zu meiner Assistentenzeit, als die ersten Defibrillatoren zum Einsatz kamen und die Intubationstechnik nicht nur Anästhesisten beigebracht wurde, gab es sehr unterschiedliche Haltungen: Ein Oberarzt liess bei akutem Herzkreislaufversagen ausnahmslos und jederzeit alle Patienten reanimieren, ganz unabhängig von Alter, Vorzustand, Prognose und sozialem Umfeld. Damit ersparte er sich Diskussionen und schwierige Entscheide, verhalf (leider nur selten) $\mathrm{zu}$ verlängertem Leben von unterschiedlicher Qualität, prolongierte aber oft ein unausweichliches Sterben und den damit verbundenen intensiv-medizinischen Aufwand. Er konnte viele Angehörige glaubhaft damit trösten, dass alles therapeutisch Mögliche getan worden sei, hatte aber auch groteske Szenen zu verantworten, wie sie Bernard Lown anlässlich der unerwünschten Reanimationsversuche bei seiner 96jährigen Mutter mit ansehen musste [1].

Der zweite Oberarzt entschied von Fall zu Fall nach bestem Wissen und Gewissen, was ihn nicht vor kollegialem Verdacht oder gelegentlichem Selbstvorwurf schützte, er habe nur nach Lust und Laune gehandelt oder situativem Druck nachgegeben. Andererseits wurde es von vielen Familien geschätzt, dass Todkranke oder Hochbetagte in hoffnungslosen Situationen ohne die traumatisierende kardiopulmonale Reanimation (CPR) in Ruhe sterben durften. Das behandelnde Team fühlte sich entlastet, ungerechtfertigte Kosten wurden eingespart.

Selbst in kaderärztliche Verantwortung eingebunden, fand ich es immer wieder sehr schwierig, die Frage «Reanimation ja oder nein?» so frühzeitig zu beantworten, wie sie zuerst nur nach notfallmässigen Aufnahmen oder in präterminalen Phasen, bald aber routinemässig bei jedem Spitaleintritt zwecks Eintrag im Kardex gestellt wurde - für den Fall eines Falles. Auch wenn die miserable Prognose klar schien, fehlten meistens wichtige Informationen und die Wünsche des Patienten oder seiner Angehörigen waren nicht bekannt. Zudem gab es sowohl bei den Behandelten wie auch im Team der Behandelnden aggressive Falken und handlungsscheue Tauben, und oft genug flatterten beide Vögel in der eigenen Brust. Der unbefriedigende, schlecht begründbare Kompromiss bestand darin, auf apparative Einsätze zu verzichten, sonst aber alle Therapien uneingeschränkt fortzuführen. Die strikte Verordnung «keine REA» hingegen brachte trotz aller gegenteiligen Beteuerungen die Gefahr mit sich, dass Patienten vernachlässigt wurden und nicht mehr die gebührende Beachtung und Betreuung erhielten, obwohl einige durchaus die Chance hatten, das Spital nochmals lebend zu verlassen. Auch der Vermerk «therapia minima» konnte als Freipass für eine gedrosselte Zuwendung missverstanden werden. Gerber [2] schlägt deshalb den Begriff «maximale Komforttherapie» vor, andere gebrauchen das Kürzel PC für genau umschriebene Palliative Care.

Reanimationen werden gerne zur Erhöhung der Spannung in Kriminalfilmen oder Ärzteserien eingesetzt. Mit Blaulicht und Martinshorn rasen die Ambulanzen unverzüglich herbei, in knatternden Helikoptern schweben Rettungssanitäter vom Himmel herab und bringen die Verblichenen mit wenigen Handgriffen und Elektroschlägen zum Wiedererblühen. Die Verfügbarkeit und Erfolgsrate der CPR ist in TV-Darstellungen unrealistisch hoch, Versagen oder Komplikationen werden selten gezeigt, weshalb die Zuschauer den falschen Eindruck erhalten, man könne und müsse immer blitzschnell helfen. Von dieser Meinung lassen sie sich bis ins hohe Alter nicht abbringen, zeigen doch Befragungen bei Senioren, dass sie, auch wenn sie über 90jährig wären, die minime Chance einer Wiederbelebung nicht ungenützt lassen möchten. Gleichzeitig fürchten sie sich aber vor einem unwürdigen Sterben an Schläuchen und Kabeln, dessen übersteigerten Horror sie ebenfalls in den Medien mitbekommen haben.

Mit diesen Bildern im Kopf, besorgten oder drängenden Angehörigen hinter sich, einem oft widersprüchlich kommunizierenden Behandlungsteam um und einer ungewissen Zukunft vor sich sollen Schwerkranke frei entscheiden [3]? Darf man eine Therapie zur Auswahl vorschlagen, die wahrscheinlich nicht mehr helfen würde? Wo liegt die Grenze der Nutzlosigkeit aller Bemühungen (futility), welche die Frage nach REA zur herzlosen Farce macht? Genügen $1 \%$, $5 \%$ oder $10 \%$ Chance, um noch alles zu versuchen, oder rechtfertigen sie einen «weisen Verzicht» [4]? Wer kann schon genau wissen, welche Erfolgsrate beim vorliegenden Patienten zu erwarten wäre? Trotz aller Erfahrung kann man sich in der Prognose blamabel täuschen:

«Keine REA» schrieb ich spätabends auf das Verordnungsblatt der Notfallstation, für einmal 
ohne langes Bedenken. Der bald 70jährige Magaziner hatte anlässlich eines vorangegangenen Spitalaufenthaltes unterschriftlich seinen Willen bekundet, im Falle einer nochmaligen Verschlechterung auf lebensverlängernde Massnahmen zu verzichten. Zuvor war der durch ein obstruktives Lungenemphysem invalidisierte Raucher dreimal wegen Bronchopneumonien auf der IPS behandelt worden, wobei zweimal eine apparative Beatmung während mehrerer Tage notwendig schien, die nur eine sehr langsame Entwöhnung vom Respirator zugelassen hatte.

Nun also war der Mann in anscheinend moribundem Zustand eingewiesen worden. Noch in Unkenntnis der Patientenverfügung hatte der Dienstarzt Laboruntersuchungen und ein Thoraxröntgenbild angeordnet: massivste Lungenüberblähung, disseminierte Infiltrate; katastrophale, nur teilweise kompensierte respiratorische Azidose mit einem pH von 7,11. Seinem nochmaligen Wunsch entsprechend und mit Einverständnis der Ehefrau wurde der noch knapp ansprechbare Bluebloater nur palliativ mit Morphium und Sauerstoff in hoher Dosierung versehen, eine Atemdämpfung bewusst in Kauf genommen. Auf die Frage am folgenden Morgenrapport, ob der Patient habe sterben können, berichtete die Stationsärztin mit einiger Verlegenheit, dass der von allen «abgeschriebene» Herr K. hochaufgerichtet im Bett sitze und soeben energisch ein Frühstück verlangt habe.

Jeder spontane Patientenwunsch bezüglich REA ist unbedingt zu respektieren. Die erstrebenswerte Autonomie darf aber nicht zum Automatismus einer routinemässigen Befragung der «schweigenden Mehrheit» führen, für die sich das Problem nur hypothetisch stellt. Sonst würden nicht nur zahlreiche Patienten und Angehörige in unangebrachte Verunsicherung gestürzt, sondern auch die Betreuenden überfordert. REA ja/nein kann nicht unter Zeitdruck und ohne hinreichende Begründung auf einem Formular abgehakt oder angeklickt werden.

Vor 20 Jahren wurden die ersten Entscheidungshilfen bezüglich «do-not-resuscitate orders» angeboten [5-7] und einige wegweisende Arbeiten publiziert, die seither durch viele Diskussionsbeiträge ergänzt worden sind, von denen im Literaturverzeichnis nur wenige Beispiele erwähnt sind [8-12]. Mit den geplanten medizinisch-ethischen Richtlinien der SAMW für DNAR-Verordnungen möchte man die Prozeduren verbessern und die Patientenautonomie sichern. Das ist zu begrüssen. Es wäre aber verfehlt, für jede Situation verbindliche Regeln zu erwarten. Nicht jeder Rest ärztlicher Verantwortung lässt sich auf vorfabrizierte Checklisten oder Entscheidungsschaltpläne auslagern.

«Darin liegt ja gerade das Kennzeichen des Gebildeten, dass er Genauigkeit in jeder Art von Wissenschaft nur soweit erwarten wird, als die Natur des Gegenstandes sie ermöglicht. Alle Ausführungen über Fragen des Handelns müssen immer etwas Skizzenhaftes haben. Man kann von ihnen keine mathematische Exaktheit verlangen. Auf dem Gebiete des Handelns und des Zweckmässigen gibt es keine festen Normen, auch nicht in der Gesundheitslehre. Was im rechten Augenblick zu tun ist, das muss der Handelnde, ähnlich wie der Arzt und der Steuermann, immer selbst sehen.» (Aristoteles, Nikomachische Ethik)

\section{Literatur}

1 Lown B. Die verlorene Kunst des Heilens. Stuttgart: Suhrkamp Taschenbuch; 2004.

2 Gerber A. Neue SAMW-Richtlinien «DNAR». Komplexe Entscheide, schwierige Umsetzung. Schweiz Ärztezeitung 2006;87(21):940-1.

3 Orentlicher D. The illusion of patient choice in end-of-life decisions. JAMA 1992;267:2101-4.

4 Alpers A. When is CPR futile? JAMA 1995; 273:156-8.

5 Council on Ethical and Judical Affairs, American Medical Association. Guidelines for the appropriate use of do-not-resuscitate orders. (1987) update. JAMA1991;265:1868-71

6 Youngner SJ, Lewandowsky W, McClish DK, et al. «Do not resuscitate» orders. Incidence and implications in a medical intensive care unit. JAMA 1985;253:54-7.

7 Charlson ME, Sax FL, MacKenzie R, et al. Resuscitation: how do we decide? A prospective study of physicians' preferences and the clinical course of hospitalized patients. JAMA 1986;255:1316-22.

8 Tomlinson T, Brody H. Futility and the ethics of resuscitation. JAMA 1990;264:1276-80.

9 Sulmasy DP, Geller G, Faden R, Levine DM. The quality of mercy. Caring for patients with «do not resuscitate» orders. JAMA 1992;267:682-6.

10 Murphy DJ, Burrows D, Santilli S, et al. The influence of the probability of survival on patients' preferences regarding cardiopulmonary resuscitation. N Engl J Med 1994;330:545-9.

11 Curtis JR, Park DR, Krone MR, Pearlman RA. Use of the medical futility rationale in do-not-attemptresuscitation orders. JAMA 1995;273:124-8.

12 Goold SD, Williams B, Arnold RM. Conflicts regarding decisions to limit treatment. A differential diagnosis. JAMA 2000;283:909-14. 